\title{
The influence of cloud top variability from radar measurements on 3-D radiative transfer
}

\author{
F. Richter ${ }^{1}$, K. Barfus ${ }^{1}$, F. H. Berger ${ }^{1,2}$, and U. Görsdorf ${ }^{2}$ \\ ${ }^{1}$ TU Dresden, Faculty of Forest, Geo and Hydro Sciences, Institute of Hydrology and Meteorology, Dresden, Germany \\ ${ }^{2}$ German Meteorological Service, Lindenberg, Germany
}

Received: 16 May 2007 - Published in Atmos. Chem. Phys. Discuss.: 11 June 2007

Revised: 6 September 2007 - Accepted: 12 September 2007 - Published: 14 September 2007

\begin{abstract}
In radiative transfer simulations the simplification of cloud top structure by homogeneous assumptions can cause mistakes in comparison to realistic heterogeneous cloud top structures. This paper examines the influence of cloud top heterogeneity on the radiation at the top of the atmosphere. The use of cloud top measurements with a high temporal resolution allows the analysis of small spatial cloud top heterogeneities by using the frozen turbulence assumption for the time - space conversion. Radiative observations are often based on satellite measurements, whereas small spatial structures are not considered in such treatments. A spectral analysis of the cloud top measurements showed slopes of power spectra between -1.8 and -2.0 , these values are larger than the spectra of $-5 / 3$ which is often applied to generate cloud field variability. The comparison of 3-D radiative transfer results from cloud fields with homogeneous and heterogeneous tops has been done for a single wavelength of $0.6 \mu \mathrm{m}$. The radiative transfer calculations result in lower albedos for heterogeneous cloud tops. The differences of albedos between heterogeneous and homogeneous cloud top decrease with increasing solar zenith angle. The influence of cloud top variability on radiances is shown. The reflectances for heterogeneous tops are explicitly larger in forward direction, in backward direction lower. The largest difference of the mean reflectances (mean over cloud field) between homogeneous and heterogeneous cloud top is approximately 0.3 , which is $30 \%$ of illumination.
\end{abstract}

\section{Introduction}

The importance of clouds in the climate system is unquestioned, because they strongly influence the insolation, the most significant energy source for the climate system.

Correspondence to: F. Richter

(falk.richter@awi.de)
Clouds are spatially highly inhomogeneous, which is determined by variations in cloud microphysics and cloud geometry. Up to now satellite measurements are not able to gauge cloud describing parameters in a spatially adequate resolution, neither for micrometeorological parameters nor for geometrical ones. But these variabilities, in the so called "subpixel" scale, strongly influence the radiative transfer. Already Randall et al. (2003) showed the correlation of smaller and larger scale behaviour of the atmospheric system. Especially in the field of radiation calculations in global atmospheric models Randall et al. (2003) adduced, that the parameterisation of the input parameters like phase, shape, and size of cloud particles but also cloud geometry is the main reason for inaccuracies of radiative transfer results. To overcome these deficiencies subgrid cloud variability is either determined by stochastic cloud generators Räisänen et al. (2004) or embedded cloud resolving models Randall et al. (2003) driven by data of the atmospheric circulation model.

Many studies have used stochastic cloud fields to investigate the influence of variabilities of macro- and microphysical parameters on radiative transfer. In most cases the variabilities have been attributed to variations in volume extinction coefficient whereas cloud geometry has been kept constant (e.g. Barker and Davies, 1992; Marshak et al., 1995a,b). Already Loeb et al. (1998) and Loeb and Coakley (1998) revealed that the cloud top structure may also have substantial effects on the radiative transfer. So the influence of cloud variability cannot be explained by assuming variations only in cloud microphysics, keeping cloud geometry, especially cloud top height, constant. Already a look at the sky reveals that the assumptions of flat cloud bottoms or tops are inappropriate even for stratiform clouds.

Therefore, the main purpose of this paper is to investigate the influence of cloud top variability on radiative transfer. This task should be done by describing the clouds as realistic as possible. But at the same time the cloud top variability has to be the only cause of differences in radiative transfer

Published by Copernicus Publications on behalf of the European Geosciences Union. 
Table 1. Cloudfield parameters.

\begin{tabular}{cccc}
\hline & cloud 1 & cloud 2 & cloud 3 \\
\hline CB [m] & 2200 & 1500 & 2800 \\
mean CT [m] & 3775.4 & 3623.3 & 3382.0 \\
std. deviation CT [m] & 135.4 & 178.8 & 132.1 \\
slope CT & -1.987 & -1.832 & -2.009 \\
temperature CT [ $\left.{ }^{\circ} \mathrm{K}\right]$ & 263 & 263 & 265 \\
temperature CB [ $\left.{ }^{\circ} \mathrm{K}\right]$ & 270 & 278 & 269 \\
pressure CB $[\mathrm{hPa}]$ & 783.5 & 852.5 & 722.5
\end{tabular}

results. In this study a full 3-D radiative transfer calculation is performed using a Monte Carlo algorithm.

To describe the variability of the cloud top no constant value, like $-5 / 3$ for the slope of the power spectrum, is implemented. Instead high-resolution radar and ceilometer measurements are used to derive the variability especially from cloud top. Atmospheric parameters used in this study like wind, temperature, and pressure have been recorded simultaneously at the Meteorological Observatory Lindenberg.

\section{Methodology}

\subsection{Simulation of cloud fields}

In this study measurements of three clouds are chosen to simulate the cloud fields for radiative transfer calculations. All clouds are assumed to consist completely of liquid water. The first ice particles in super cooled clouds appear at temperatures between $263^{\circ} \mathrm{K}$ and $258^{\circ} \mathrm{K}$ (Lamb, 2002). The cloud top temperature of all three selected clouds are above $263^{\circ} \mathrm{K}$. In Table 1 the simulated cloud fields are characterised $(\mathrm{CT}=$ cloud top, $\mathrm{CB}=$ cloud base $)$.

The cloud types have been chosen to cover a great part of the natural diversity of geometrical cloud characteristic. Cloud 1 is the type geometrical thick cloud with variable top, cloud 2 geometrical thick with less variable cloud top and cloud 3 represents a geometrical thin cloud with variable top. The fourth type a thin cloud with homogeneous top is not considered, because there was no fitting measurements of radar and additional data available. For these three measured clouds the following way of simulating cloud fields for radiative transfer calculations is performed. The time to space transformation of cloud top heights, measured by vertical pointing radar is based on the so called "frozen turbulence assumption", which assumes no changes of the cloud field during the measurements.

To generate the 2-D cloud top field from 1-D measurement data, the iterative amplitude adjusted Fourier transform (IAAFT) algorithm developed by Schreiber and Schmitz (1996, 2000) was applied. This method is based on the application of Fourier spectra to characterise two point statistics of spatial or temporal data. Fourier methods have been used widely in previous studies for cloud modelling (e.g. Barker and Davies, 1992). Using the IAAFT algorithm the step from a one-dimensional time series to a two-dimensional data field has been done. The improvement after applying the IAAFT is that the simulated field and the measured time series of cloud top height are equal in power spectrum and the amplitude distribution, respectively. From a measured time series, $s_{n}$ (with $n$ is time or space) with $N$ values, the power spectrum $S_{k}$ (with $k$ wave numbers) is calculated as

$S_{k}^{2}=\left|\sum_{n} s_{n} e^{\frac{i 2 \pi k n}{N}}\right|^{2}$.

The relevant value describing the variability of the time series is the slope of a power law regression of the power spectrum and the corresponding wave numbers. A straight line continuation of the slope in the scope of higher frequencies is dependent on the absence of scale breaks in the power spectrum. Furthermore a sorted list of the measured values $s_{n}$ is necessary for the IAAFT algorithm. The iteration starts with a random shuffle of $s_{n}$. The first two steps of the algorithm are the adjustment of 1) the Fourier coefficients and 2) the amplitudes. To achieve the desired power spectrum the Fourier transform of the time series is calculated in each iteration. The absolute values of the coefficients are replaced by those from the measured time series while the phases are retained. A backward transform of these coefficients would produce an amplitude distribution which is not the same as the measured one. Therefore the second step is the adjustment of the amplitude distribution, where the amplitudes are sorted and replaced by the sorted values of the original values. These two steps of the iteration have to be repeated until the power spectrum and the amplitude distribution of generated and measured values are matching in sufficient conditions.

The derivation of a 2-D variability grid from a 1-D spectrum with the assumption of isotropic statistics leads to an underestimation of the variance of the 2-D field. This means that the slope of a single row of the 2-D field is much lower then the slope of the 1-D time series. This problem is discussed in Austin et al. (1994), and they propose to use

$\gamma=\beta-1$

where $\beta$ is the slope of the 1-D spectrum of the measured time series and $\gamma$ is the spectrum that produces a 2-D field consisting of the 1-D value $\beta$. In other words, a backward transform of the 2-D Fourier coefficients derived from $\gamma$ yields in a field where the mean slope of the power spectra of every row and column is around $\beta$.

So, cloud top fields were generated consisting of the same power spectrum and amplitude distribution as the 1-D measured time series of cloud top height.

The vertical resolution due to the measurements is assigned to the 2-D field by the IAAFT. To get a higher vertical 


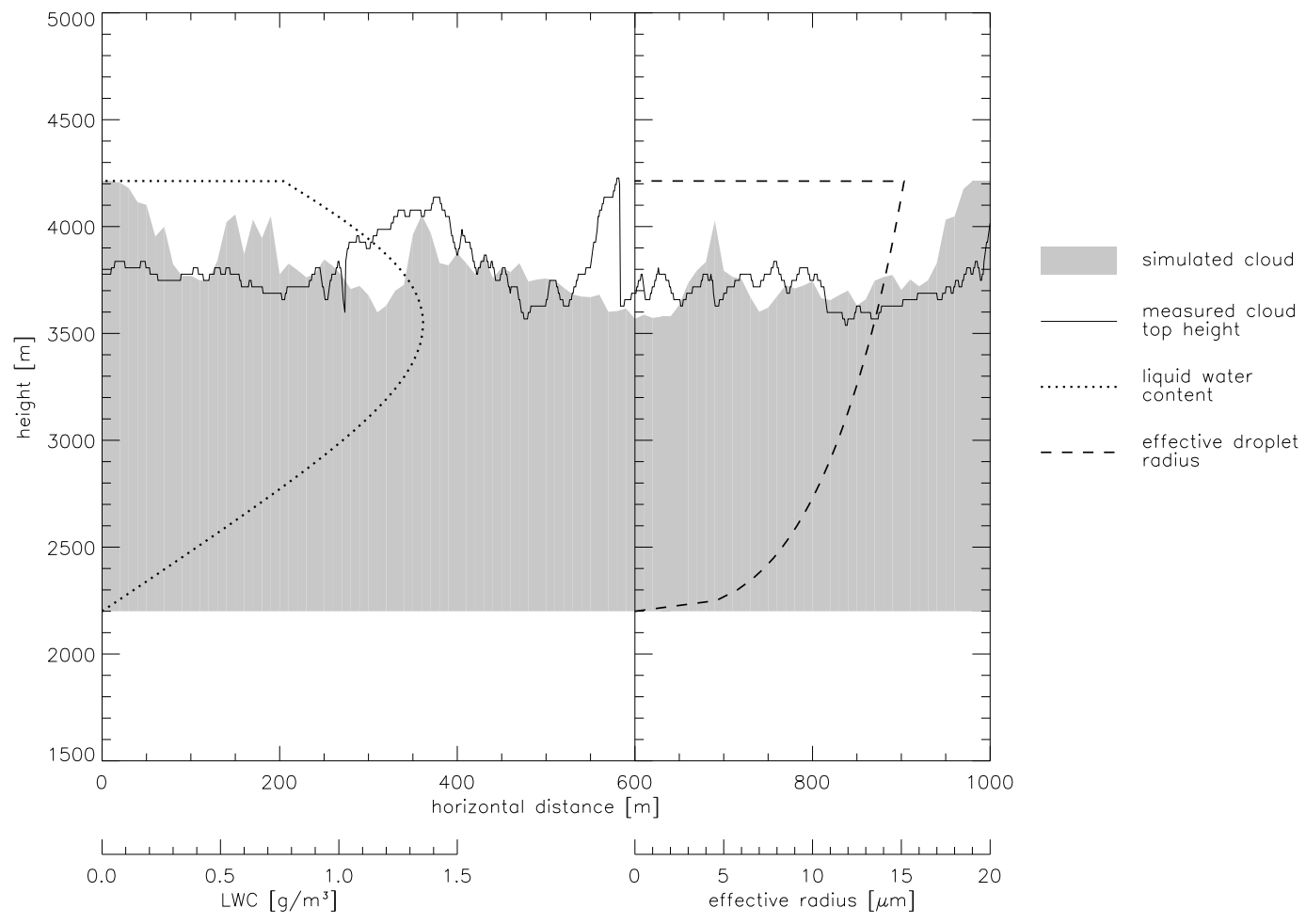

Fig. 1. Measured and simulated cloud top height, subadiabatic LWC profile and adiabatic profile of the effective radius.

resolution of cloud tops for the 3-D cloud field, a linear interpolation of the cumulative amplitude distribution is performed. So the second step of the IAAFT, the amplitude adjustment, is done using a refined amplitude distribution.

The vertical dimension of the cloud field is characterised by a subadiabatic liquid water content (LWC) and an adiabatic profile of the effective radius. The LWC profile is based on the study of Chin et al. (2000). In this study a weighting function is applied to describe the subadiabatic character of the profile. This weighting function is given by

$$
f(\hat{z})=\exp \left(-\alpha \cdot \hat{z}^{\beta}\right)
$$

where $\hat{z}$ is the scaled height within the cloud and $\alpha$ and $\beta$ are positive constants. In the study Chin et al. (2000) give two types of weighting functions: one is related to subadiabatic conditions involving cloud top entrainment alone and the other considers both cloud top entrainment and drizzle effects. To ensure the validity of Mie theory for calculation of optical properties the first type was chosen, with the parameterisation of $\alpha=1.375$ and $\beta=4$. The value of $\alpha$ is recommended by Chin et al. (2000) and with $\beta=4$ a strong cloud top entrainment is simulated. The weighting function given above weights an adiabatic $\mathrm{LWC}\left(\mathrm{LWC}_{\mathrm{ad}}\right)$ profile to a subadiabatic one $\left(\mathrm{LWC}_{\text {subad }}\right)$ in the following way

$\operatorname{LWC}_{\text {subad }}(\hat{z})=\operatorname{LWC}_{\mathrm{ad}}(\hat{z}) \cdot f(\hat{z})$
The adiabatic LWC profile and the weighting function are calculated from cloud base to the highest cloud top. Then the accordant values for the discretised heights are interpolated and allocated to the overall cloud level.

The adiabatic profile of the effective radius is calculated using the study of Brenguier et al. (2000). The way of calculation is the following,

$\operatorname{LWC}_{\mathrm{ad}}(h)=C_{w} \cdot h$,

$r_{v_{a d}}(h)=(A \cdot h)^{\frac{1}{3}} \cdot N_{a d}^{-\frac{1}{3}}$,

with: $A=\frac{C_{w}}{\frac{4}{3} \cdot \pi \cdot \rho_{w}}$,

$$
\begin{aligned}
r_{e_{a d}}(h) & =k^{-\frac{1}{3}} \cdot r_{v_{a d}} \\
& =(A \cdot h)^{\frac{1}{3}} \cdot\left(k \cdot N_{a d}\right)^{-\frac{1}{3}} \text { and } \\
r_{s_{a d}}= & k^{\frac{1}{6}} \cdot r_{v_{a d}}
\end{aligned}
$$

Here, $C_{w}$ is the moist adiabatic condensate coefficient, $h$ is the altitude above cloud base, $\rho_{w}$ the liquid water density, $r_{v}$ the mean volume radius, $r_{e}$ the droplet effective radius and $r_{s}$ the mean surface radius of the droplet size distribution. The parameter $k$ relates $r_{v}$ and $r_{e}$ and $N$ is the droplet number concentration in the cloud. The subscript " $a d$ " for $N, r_{e}, r_{v}$ and $r_{s}$ refers to the adiabatic values. According to Brenguier 


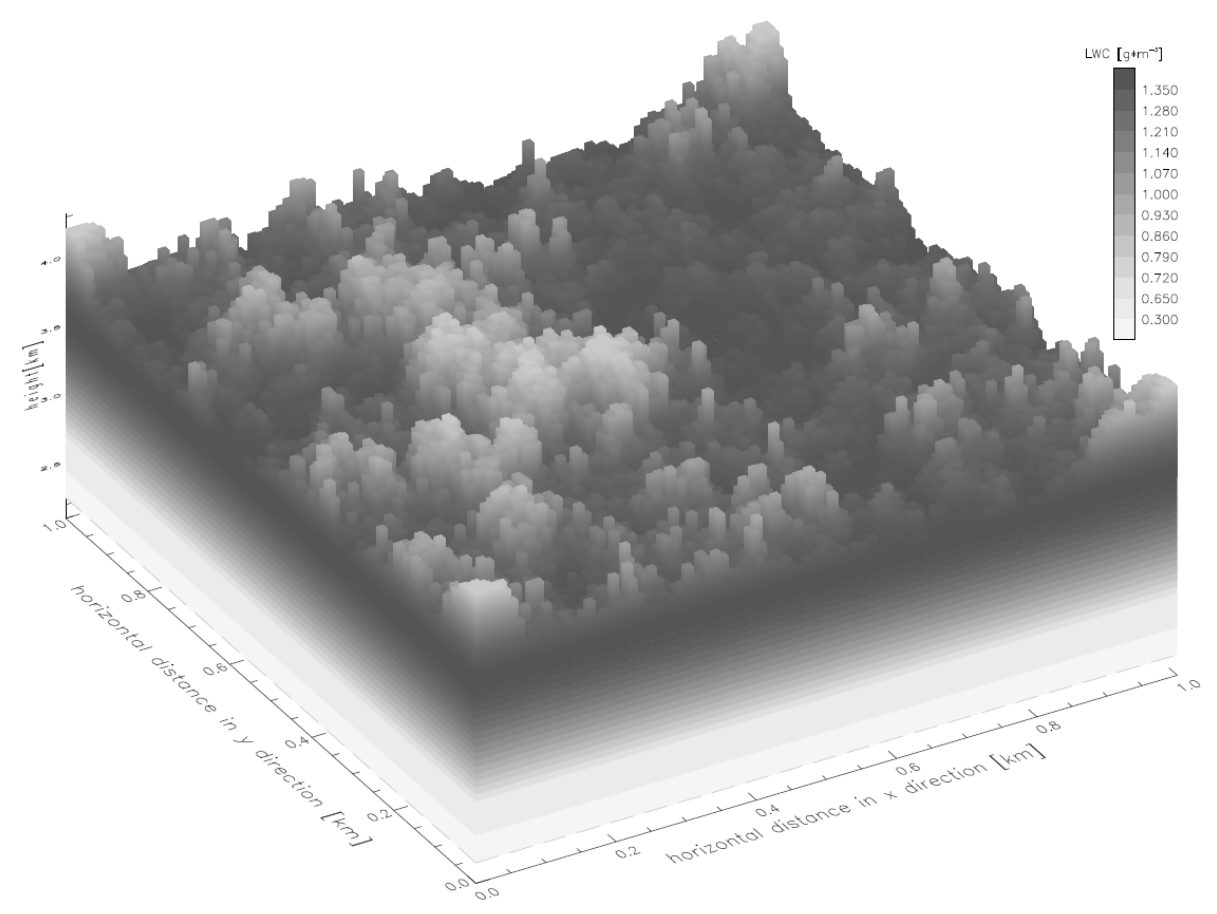

Fig. 2. LWC of the 3-D simulated cloud field.

et al. (2000) $k$ is set to 0.67 for continental air masses and $N_{a d}$ is $250 \mathrm{~cm}^{-3}$ representing polluted air.

This combination of a non-adiabatic LWC and an adiabatic profile of the effective radius is corresponding to the term of "inhomogeneous mixing", mentioned in Baker et al. (1980). This mixing scheme takes place when the time of evaporation of a droplet with radius $r$ is smaller than the time for the complete mixing process in the layer. In this case all droplet-radii in the volume affected by entrainment completely evaporate.

Figure 1 shows measured time series of cloud top height, a slice of geometrical properties of the simulated cloud field, and profiles of LWC and effective radius. Figure 2 illustrates the three-dimensional cloud field based on these data. As the counterpart to the cloud field with heterogeneous top a field with homogeneous cloud top has been generated using the mean cloud top height of the measured data.

\subsection{Monte Carlo simulations}

Monte Carlo simulations are performed with the model MCUNIK, described in Macke et al. (1999). The model assumes periodic boundary conditions in $\mathrm{x}$ and $\mathrm{y}$-direction. Each simulation runs with $10^{6}$ photons, which are uniformly released at the top of the domain. The Monte Carlo model is equipped with the local estimate approch for example described by Barker et al. (2003). This approach enables to calculate reflectances with a smaller amount of injected photons by tracking secondary photons released on every scattering event on there direct way to the detector.
The solar zenith angle is set to $0^{\circ}, 30^{\circ}$ and $60^{\circ}$, the solar azimuth angle is constant at $0^{\circ}$; observation angles are $0^{\circ}$, $30^{\circ}$ and $60^{\circ}$ for zenith angle and $0^{\circ}, 60^{\circ}, 120^{\circ}$ and $180^{\circ}$ for azimuth angle, respectively. Cloud optical properties, like volume extinction coefficent, single scattering albedo, and phase function are calculated by Mie theorie for a wavelength of $0.6 \mu \mathrm{m}$ assuming a modified gamma distribution for cloud droplet sizes.

Outside the cloudy regions Rayleigh scattering has been applied, inside the cloud Rayleigh and Mie scattering are considered. The absorption of molecules has been neglected.

The surface albedo is examined as lambertian reflection. The value is calculated from a bidirectional reflectance distribution function (BRDF) for pasture land. This albedo is also known as "white-sky" albedo (Lucht, 2000). The parameterisation of the BRDF for pasture land is taken from Rahman et al. (1993).

\section{Results}

An advantage of this study is the use of cloud top variability from radar data. In many studies, power spectra are represented via their slopes in log-log plots calculated by least squares linear regression (assuming power law behaviour). Already Loeb et al. (1998) assumed the widely used slope of $-5 / 3$ to generate cloud top fields. The analysis of the measured time series revealed that the slopes with values of -1.8 to -2.0 are always larger than $-5 / 3$ (Fig. 3). Thus lower 


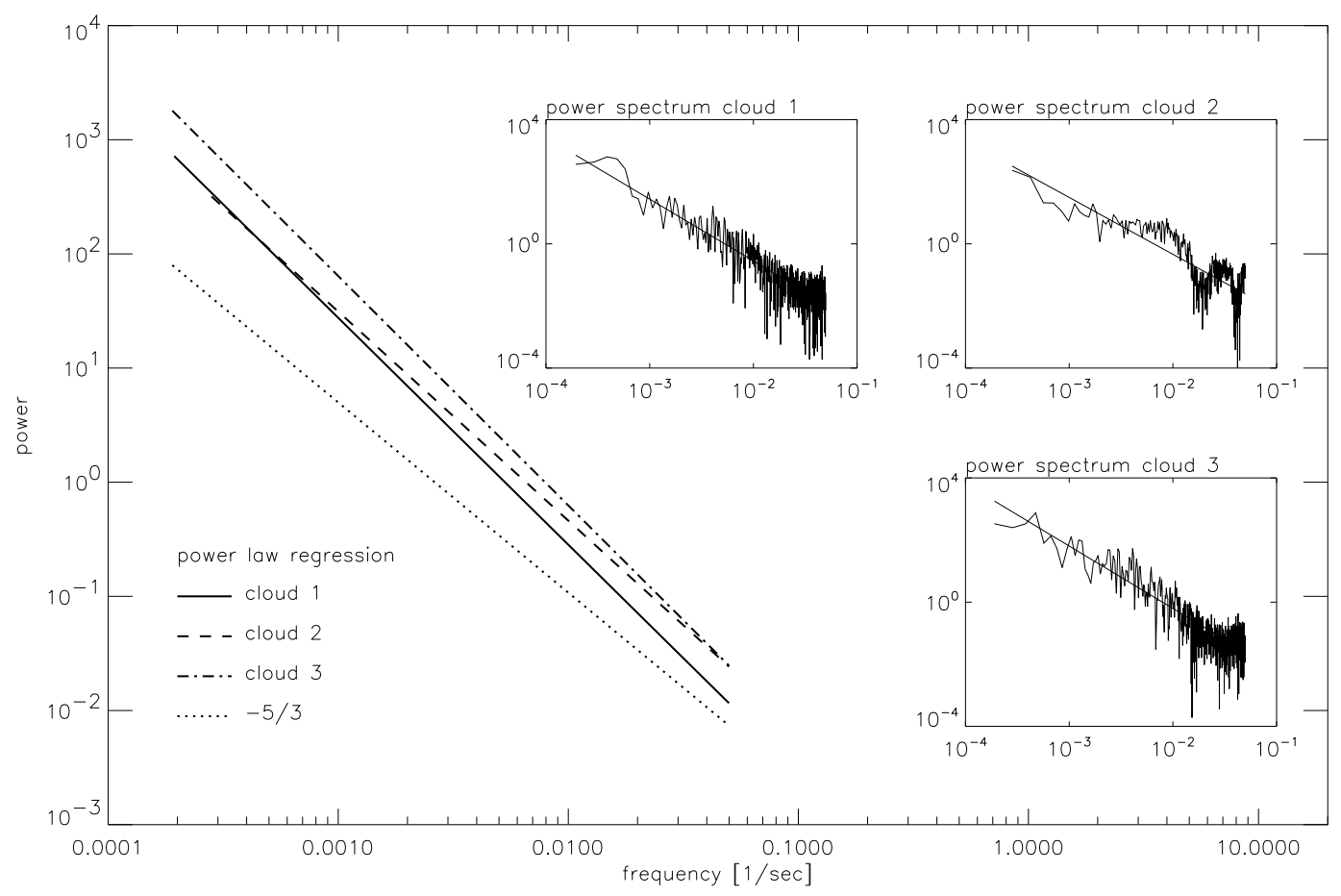

Fig. 3. Comparison of calculated power spectra.

frequencies and with them the spatially (or temporally) larger variabilities play an important role for the description of the cloud top variance. So in this study the spectra of the measured cloud top data were used to generate cloud top fields.

The focus of this study is the comparison of the radiative transfer results regarding the differences between clouds with homogeneous and heterogeneous tops. The albedo as the mean value over the whole cloud field provides a first overview. Reflectances in several directions deliver more insight. Figure 4 shows the calculated albedo values for cloud 1 and Table 2 summarises the albedo results for all three clouds. The calculated difference is defined as heterogeneous albedo minus homogeneous one. Here the albedo for heterogeneous cloud top is lower in most cases and increases with increasing solar zenith angle $\left(\theta_{\text {sun }}\right)$. The largest difference is about $1.1 \%$.

Figure 4 indicates that besides cloud top variability also the illumination angle (here only changes in solar zenith angles) influences the albedo.

There are higher albedo values with increasing solar zenith angle $\left(\theta_{\text {sun }}\right)$, whereas the differences between homogeneous and heterogeneous cloud top are decreasing. According to the one-dimensional radiative transfer effect (Varnai and Davies, 1999) one reason of the albedo increase with increasing $\theta_{\text {sun }}$ is that cloud particles scatter light preferable in forward direction, whereby for overhead sun the solar radiation penetrates deeper into the cloud. This behaviour is well illustrated by the comparison of the photons' penetration depth of
Table 2. Calculated albedo values [\%] for the different cloud fields and solar zenith angle $\left(\theta_{\text {sun }}\right)$, the difference is defined as heterogeneous minus homogeneous albedo value.

\begin{tabular}{cccc}
\hline$\theta_{\text {sun }}$ & $0^{\circ}$ & $30^{\circ}$ & $60^{\circ}$ \\
\hline \multicolumn{4}{c}{ cloud 1 } \\
\hline $\begin{array}{c}\text { homogeneous } \\
\text { heterogeneous } \\
\text { difference }\end{array}$ & 10.568 & 10.640 & 10.826 \\
\hline & -0.229 & -0.050 & 0.015 \\
\hline cloud 2 & & \\
\hline homogeneous & 10.877 & 10.912 & 11.020 \\
heterogeneous & 10.785 & 10.896 & 11.032 \\
difference & -0.092 & -0.016 & 0.012 \\
\hline & cloud 3 & & \\
\hline homogeneous & 8.587 & 8.817 & 9.472 \\
heterogeneous & 7.456 & 8.685 & 9.478 \\
difference & -1.131 & -0.132 & 0.006 \\
\hline
\end{tabular}

the different illumination angles (Figs. 5, 6 for cloud 1). The penetration depth is used as the measure for the lowest $\mathrm{z}$ position photons reach on their path through the cloud. This position mirrors the optical properties of the so far travelled path. 


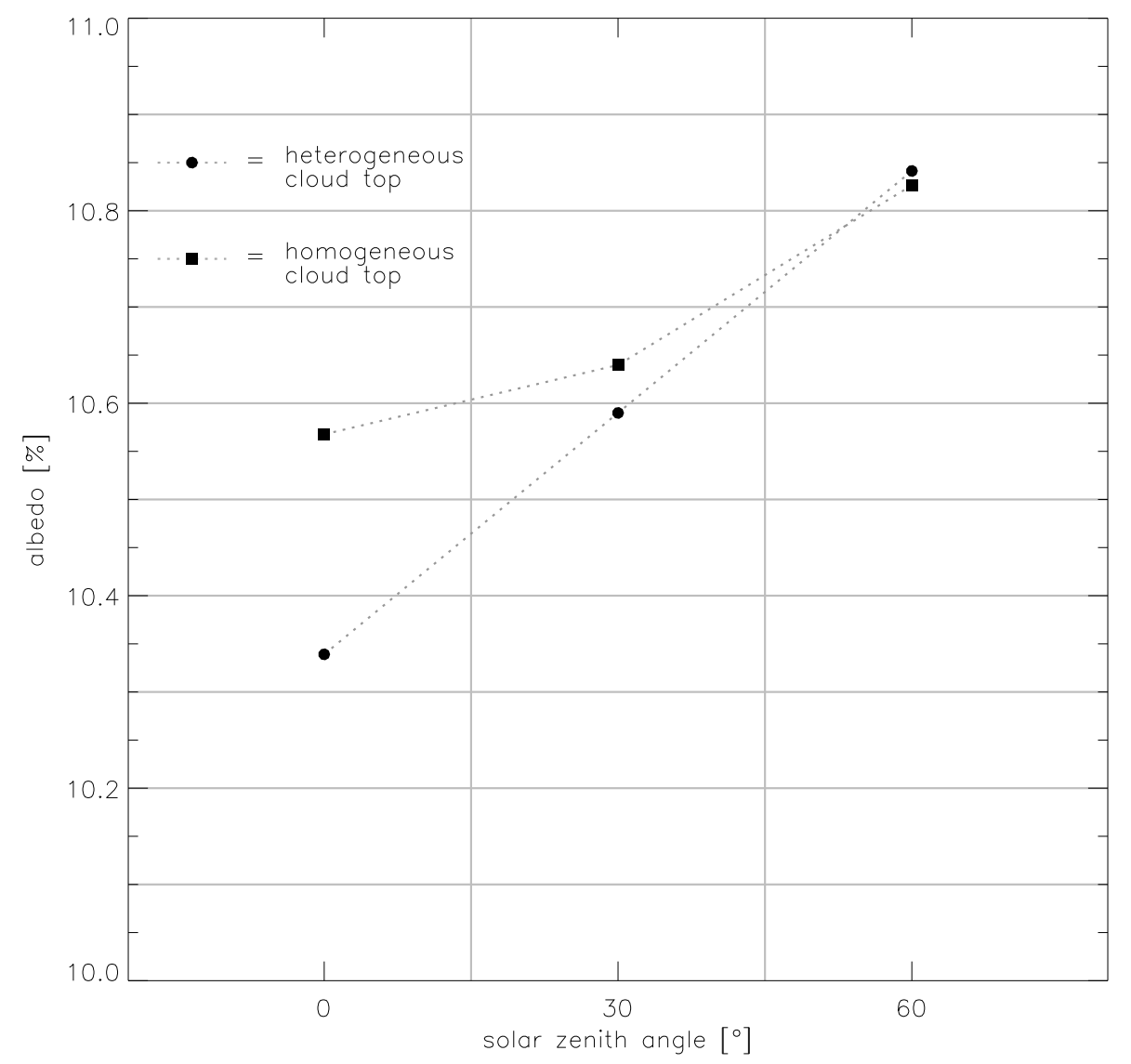

Fig. 4. Mean albedo values for cloud 1 .

Furthermore cloud fields tend to appear more homogeneous from oblique directions than from above, which enhances the albedo increase mentioned above (Varnai and Davies, 1999). The cause of the deeper penetration at heterogeneous cloud tops is the larger surface which leads to more transitions between cloudy parts and non-cloudy ones (Varnai and Davies, 1999). This added transport into the cloud is also pictured by transmission and absorption (Table 3). The simulated albedo values for cloud 2 and 3 are similar showing increasing albedo values and decreasing differences between homogeneous and heterogeneous tops with increasing $\theta_{\text {sun }}$. The significant difference between homogeneous and heterogeneous cloud top at $\theta_{\text {sun }}=0^{\circ}$ is $0.2 \%$ for cloud 1 . Cloud 2 with less variability shows only a difference of $0.1 \%$ and the thin and variable cloud 3 shows the largest difference of $1.1 \%$. The high transmission of cloud 3 with simultaneously low absorption is caused by the short vertical expansion of this cloud.

The results mentioned above already denote some aspects of the influence that cloud top variability has on radiative transfer, which is first the lower albedo of heterogeneous cloud top and second the larger penetration depth. Now the effects on reflectances are focussed. Reflectances are calcu- lated for nine observation angles, for $30^{\circ}$ and $60^{\circ}$ zenith with changes in azimuth of $0^{\circ}, 60^{\circ}, 120^{\circ}$, and $180^{\circ}$, respectively, and the direction of $0^{\circ}$ zenith and $0^{\circ}$ azimuth.

The reflectances of these observation angles are simulated for the three solar zenith angles of $0^{\circ}$ (Fig. 7), $30^{\circ}$ and $60^{\circ}$ zenith and $0^{\circ}$ azimuth.

Figure 7 shows the calculated reflectances for cloud 1 as mean values over the cloud field with corresponding minimum and maximum values. The azimuth angle of illumination is $0^{\circ}$, so the azimuth observation angle of $0^{\circ}$ is the backward direction relative to illumination, $180^{\circ}$ is forward and $60^{\circ}$ and $120^{\circ}$ are sideways, respectively. The reflectance is defined as the ratio of reflected to incident radiation. The variability of the reflectances for homogeneous cloud tops shown in Fig. 7 is the result of the uncertainty of the Monte Carlo model. These uncertainty is determined by the random nature of the Monte Carlo model and by using the local estimation approach with an obviously too low number of simulated photons.

Figures 8 and 9 show the calculated differences, defined as heterogeneous reflectance minus homogeneous one. The maximum difference of the mean reflectances between homogeneous and heterogeneous cloud top is approximately 

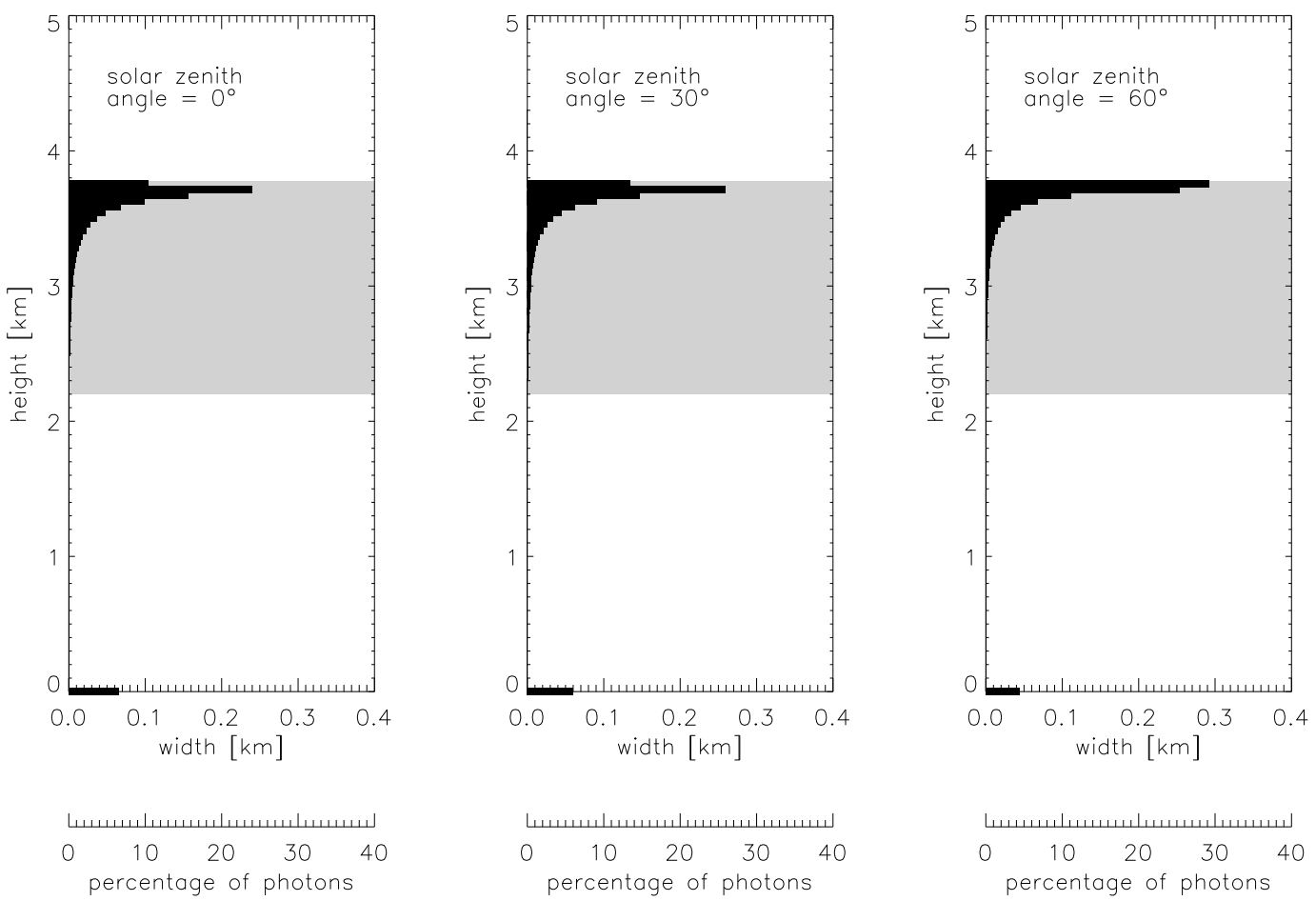

Fig. 5. Penetration depth of cloud 1 (homogeneous top) for different solar zenith angles.
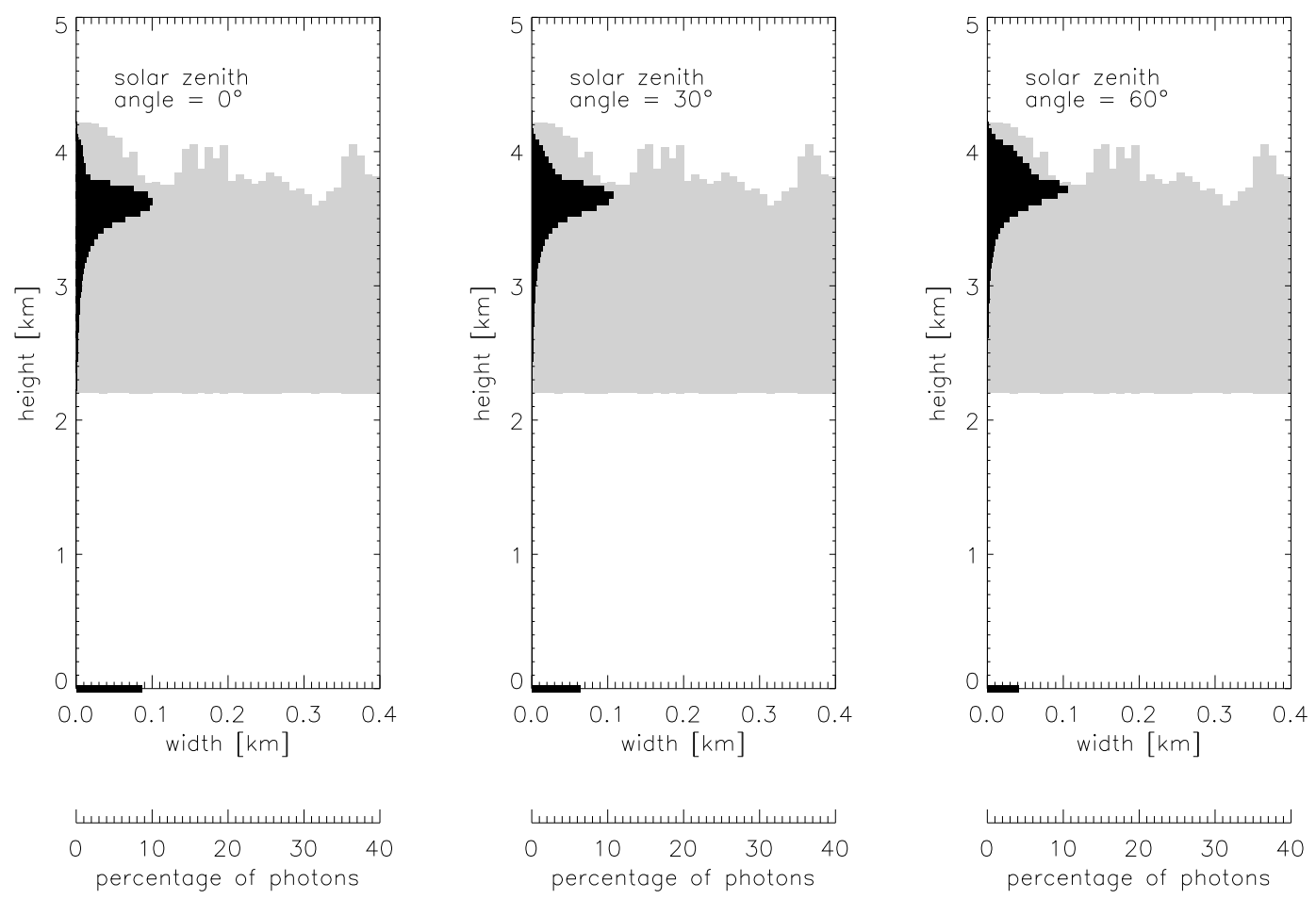

Fig. 6. Penetration depth of cloud 1 (heterogeneous top) for different solar zenith angles. 


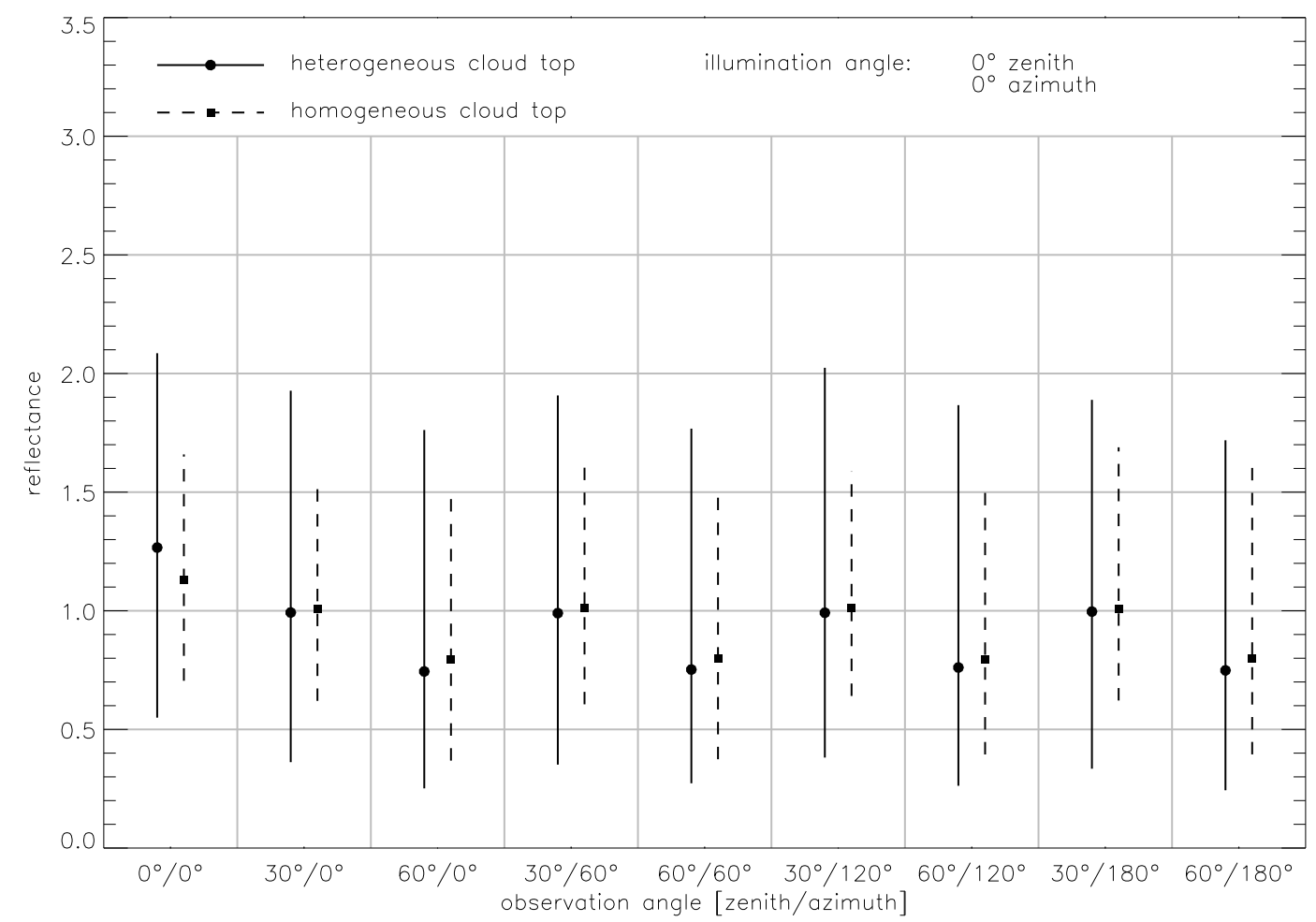

Fig. 7. Reflectances of cloud $1\left(\theta_{\text {sun }}=0^{\circ}\right)$ for different observation angles.

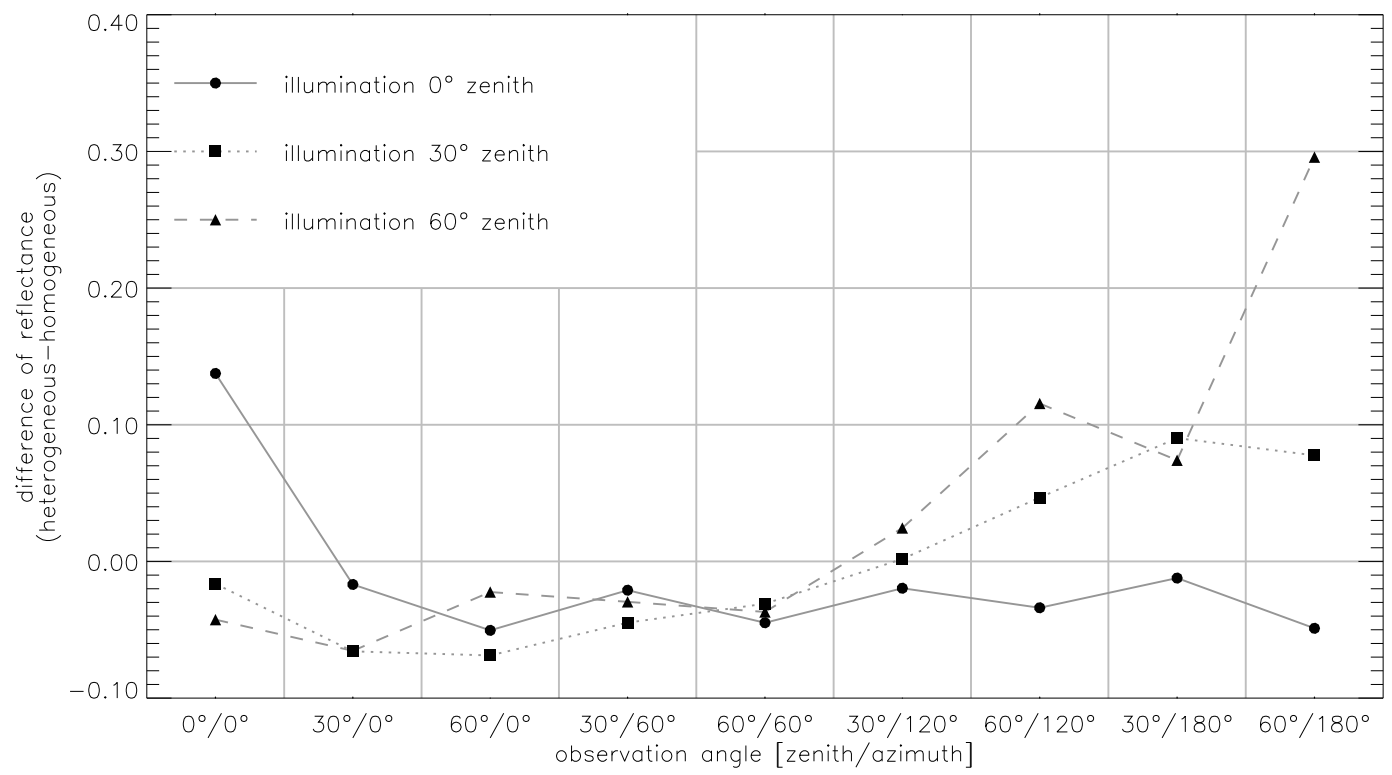

Fig. 8. Differences between heterogeneous and homogeneous reflectances for several illumination and observation angles. 

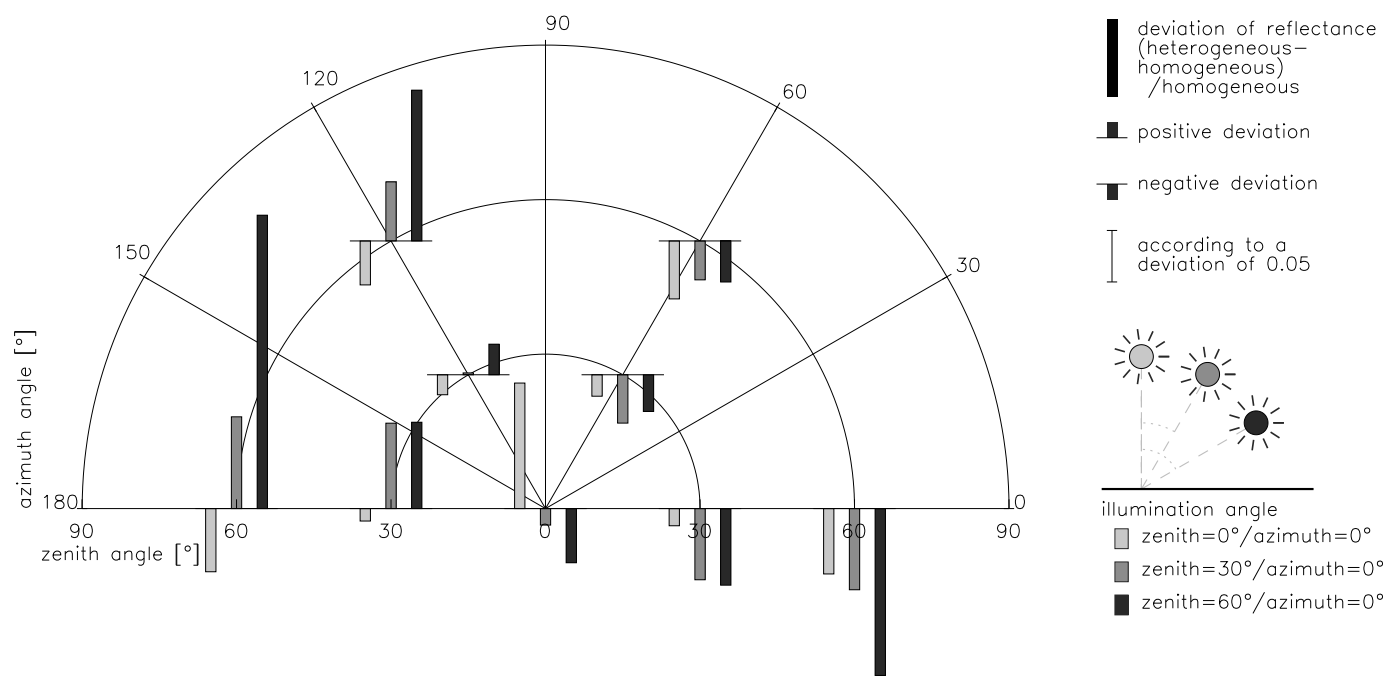

Fig. 9. Deviation of reflectance ((heterogeneous - homogeneous)/homogeneous) for several illumination and observation angles.

0.3 , which is $30 \%$ of illumination. The largest differences appear in forward and backward direction relative to illumination direction, whereas the differences have a negative maximum in backward direction (homogeneous $>$ heterogeneous) and a positive one in forward direction.

The largest negative difference is found in backward direction when the zenith angles of illumination and observation are equal. The maximum positive difference is found in forward direction for equal zenith angles of illumination and observation.

The preferred forward direction can be explained by the forward peak of the Mie phase function. The probability that a photon turns around in the backward direction is very low compared to proceeding in forward direction. As mentioned above, the cloud top heterogeneity causes a higher transport into cloudy regions, which leads to more intense scattering. The probability of a complete photon turn is much lower for clouds with heterogeneous tops. Therefore the reflectance in backward direction is lower for clouds with heterogeneous tops then for homogenous ones, which leads to the negative differences shown in the Fig. 8. The largest reflectances appear in forward direction (azimuth angle of $180^{\circ}$ ) when the zenith angles of illumination and observation are equal. This behaviour is determined by two effects. One is the dominance of the forward peak of Mie scattering, that causes on one hand the escape of the photon in forward direction and on the other hand the deeper penetration for lower solar zenith angles. The deeper penetration causes the distance the photon has to propagate back to the detector to be larger for lower zenith angles than for higher ones. This longer way is the second effect and causes a higher attenuation of the photons' energy.
Table 3. Transmission and absorption [\%] for the different cloud fields (described in Table 1) and solar zenith angles.

\begin{tabular}{|c|c|c|c|c|}
\hline & \multirow[t]{2}{*}{ variability } & \multicolumn{3}{|c|}{$\theta_{\text {sun }}$} \\
\hline & & $0^{\circ}$ & $30^{\circ}$ & $60^{\circ}$ \\
\hline \multicolumn{5}{|c|}{ transmission } \\
\hline \multirow[t]{2}{*}{ cloud 1} & homogeneous & 6.6920 & 6.1150 & 4.5183 \\
\hline & heterogeneous & 8.8190 & 6.5252 & 4.2626 \\
\hline \multirow[t]{2}{*}{ cloud 2} & homogeneous & 3.8751 & 3.5504 & 2.6138 \\
\hline & heterogeneous & 4.5957 & 3.6744 & 2.5222 \\
\hline \multirow[t]{2}{*}{ cloud 3} & homogeneous & 24.9300 & 22.8276 & 16.8455 \\
\hline & heterogeneous & 35.1987 & 24.0418 & 16.8273 \\
\hline \multicolumn{5}{|c|}{ absorption } \\
\hline \multirow[t]{2}{*}{ cloud 1} & homogeneous & 0.1385 & 0.1276 & 0.0963 \\
\hline & heterogeneous & 0.1705 & 0.1329 & 0.0937 \\
\hline \multirow[t]{2}{*}{ cloud 2} & homogeneous & 0.2619 & 0.2409 & 0.1795 \\
\hline & heterogeneous & 0.3039 & 0.2471 & 0.1750 \\
\hline \multirow[t]{2}{*}{ cloud 3} & homogeneous & 0.0203 & 0.0192 & 0.0155 \\
\hline & heterogeneous & 0.0219 & 0.0199 & 0.0162 \\
\hline
\end{tabular}

\section{Conclusions}

Although earlier studies examined the influence of cloud top variability on radiation, only a few have used measured data with high resolution. Several of the applied techniques have been used in earlier studies, but not necessarily in this way. This study combines them and therefore tries to describe clouds as realistic as possible, always keeping in mind that cloud top variability has to be the only cause of differences in radiative transfer results. The present study should extend 
the earlier studies that deal with cloud top heterogeneity effects on radiative transfer in cloudy atmosphere in general.

The Fourier analysis of the measured time series of cloud top height shows that the calculated slopes of the power spectrum are larger than the widely used assumption of $-5 / 3$ owning values of -1.8 to -2.0 . So the spatial small-scale variability seems not that important to describe the variance of a cloud top.

The examined cases show an increase of the differences between homogeneous and heterogeneous albedo values at larger cloud top variability. Convective clouds with often larger geometrical cloud top variability are therewith more effected by the influence of these heterogeneity effects than stratiform clouds for example. The solar zenith angle has a larger influence on radiative transfer than cloud top variability, but for large solar zenith angles the differences of albedo values are negligible.

The differences of reflectances are also larger for higher solar zenith angles, so the neagtive maximum (homogeneous $>$ heterogeneous) appears in backward direction and the positve maximum in forward direction. The largest difference of the mean reflectances between homogeneous and heterogeneous cloud top is approximately 0.3 , so it can be important for measurements in these directions. Many atmospheric parameters are deviated from satellite measurements. The radiances are gauged at the VIS and IR spectra. The influence of cloud top variability on radiative transfer is not the most important one of course, but might have influence on the accuracy of deviated parameters.

Acknowledgements. The institute of meteorology at the Technische Universität Dresden with C. Bernhofer and all members is gratefully acknowledged for managing the working environment. Special thanks to A. Schwiebus for proof-reading. This paper is based on the master thesis of F. Richter.

Edited by: V. Fomichev

\section{References}

Austin, R., England, A., and Wakefield, G.: Special problems in the estimation of power-law spectra as applied to topographical modeling, Geoscience and Remote Sensing, IEEE Transactions on, 32, 928-939, 1994.

Baker, M., Corbin, R. G., and Latham, J.: The influence of entrainment on the evolution of cloud droplet spectra: I A model of inhomogeneous mixing, Q. J. Roy. Meteor. Soc., 106, 581-598, 1980.

Barker, H. W. and Davies, J. A.: Solar Radiative Fluxes for Stochastic, Scale-invariant Broken Cloud Fields, J. Atmos. Sci., 49, 1115-1126, 1992.
Barker, H. W., Goldstein, R. K., and Stevens, D. E.: Monte Carlo Simulation of Solar Reflectances for Cloudy Atmospheres, J. Atmos. Sci., 60, 1881-1894, 2003.

Brenguier, J.-L., Pawlowska, H., Schüller, L., Preusker, R., Fischer, J., and Fouquart, Y.: Radiative Properties of Boundary Layer Clouds: Droplet Effective Radius versus Number Concentration, J. Atmos. Sci., 57, 803-821, 2000.

Chin, H.-N. S., Rodriguez, D. J., Cederwall, R. T., Chuang, C. C., Grossman, A. S., Yio, J. J., Fu, Q., and Miller, M. A.: A Microphysical Retrieval Scheme for Continental Low-Level Stratiform Clouds: Impacts of the Subadiabatic Character on Microphysical Properties and Radiation Budgets, Mon. Weather Rev., 128, 2511-2527, 2000.

Lamb, D.: Encyclopedia of atmospheric sciences, Academic Press, 2002.

Loeb, N. G. and Coakley, J. A. J.: Inference of Marine Stratus Cloud Optical Depths from Satellite Measurements: Does 1D Theory Apply?, J. Climate, 11, 215-233, 1998.

Loeb, N. G., Varnai, T., and Winker, D. M.: Influence of SubpixelScale Cloud-Top Structure on Reflectances from Overcast Stratiform Cloud Layers, J. Atmos. Sci., 55, 2960-2973, 1998.

Lucht, W.: An algorithm for the retrieval of albedo from space using semiempirical BRDF models, IEEE Transactions on Geoscience and Remote Sensing, 38, 977-998, 2000.

Macke, A., Mitchell, D., and Bremen, L.: Monte Carlo Radiative Transfer Calculations for Inhomogeneous Mixed Phase Clouds, Physics and Chemistry of the Earth, Part B: Hydrology, Oceans Atmos., 24, 237-241, 1999.

Marshak, A., Davis, A., and Titov, G.: The verisimilitude of the independent pixel approximation used in cloud remote sensing, Remote Sens. Environ., 52, 71-78, 1995a.

Marshak, A., Davis, A., Wiscombe, W., and Cahalan, R.: Radiative smoothing in fractal clouds, J. Geophys. Res., 100, $26247-$ 26 262, 1995 b.

Rahman, H., Verstraete, M. M., and Pinty, B.: Coupled surfaceatmosphere reflectance (CSAR) model. 1: Model description and inversion on synthetic data, J. Geophys. Res., 98, 20 779-20 801, 1993.

Räisänen, P., Barker, W. H., Khairoutdinov Marat, F., Li, J., and Randall, A. D.: Stochastic generation of subgrid-scale cloudy columns for large-scale models, Q. J. Roy. Meteor. Soc., 130, 2047-2067, 2004.

Randall, D., Khairoutdinov, M., Arakawa, A., and Grabowski, W.: Breaking the Cloud Parameterization Deadlock, B. Am. Meteor. Soc., 84, 1547-1564, 2003.

Schreiber, T. and Schmitz, A.: Improved surrogate data for nonlinearity tests, Phys. Rev. Lett., 77, 635-638, 1996.

Schreiber, T. and Schmitz, A.: Surrogate time series, Physica D, 142, 346-382, 2000.

Varnai, T. and Davies, R.: Effects of Cloud Heterogeneities on Shortwave Radiation: Comparison of Cloud-Top Variability and Internal Heterogeneity, J. Atmos. Sci., 56, 4206-4224, 1999. 